\title{
Seismic detection of acoustic sharp features in the CoRoT target HD 49933
}

\author{
A. Mazumdar ${ }^{1}$, E. Michel ${ }^{2}$, H. M. Antia ${ }^{3}$, and S. Deheuvels ${ }^{2,4}$ \\ ${ }^{1}$ Homi Bhabha Centre for Science Education, TIFR, V. N. Purav Marg, Mankhurd, Mumbai 400088, India \\ e-mail: anwesh@tifr.res.in \\ 2 LESIA, Observatoire de Paris, UMR8109 CNRS, Université Pierre et Marie Curie, Université Denis Diderot, Place J. Janssen, \\ 92195 Meudon, France \\ 3 Tata Institute of Fundamental Research, Homi Bhabha Road, Mumbai 400005, India \\ 4 Astronomy Department, Yale University, PO Box 208101, New Haven, CT 065208101, USA
}

Received 22 November 2011 / Accepted 10 February 2012

\section{ABSTRACT}

\begin{abstract}
The technique of determining the acoustic location of layers of sharp changes in the sound speed inside a star from the oscillatory signal in its frequencies is applied to a solar-type star, the CoRoT target, HD 49933. We are able to determine the acoustic depth of the second helium ionisation zone of HD 49933 to be $794_{-68}^{+55} \mathrm{~s}$. The acoustic depth of the base of the convective zone is found to be $1855_{-412}^{+173} \mathrm{~s}$ where the large error bars reflect the ambiguity in the result, which is difficult to determine with present precision on the frequencies because of the intrinsically weak nature of the signal. The positions of both these layers are consistent with those in a representative stellar model of HD 49933.
\end{abstract}

Key words. stars: individual: HD 49933 - stars: oscillations - stars: interiors - stars: fundamental parameters

\section{Introduction}

Any localised feature in the sound speed inside a star, such as the one caused by the change in the temperature gradient at the base of the convection zone, introduces an oscillatory term in frequencies as a function of radial order $n$ that is proportional to $\sin \left(2 \tau_{\mathrm{d}} \omega_{n, \ell}+\phi\right)$, (Gough 1990) where $\tau_{\mathrm{d}}=\int_{r_{\mathrm{d}}}^{R} \frac{\mathrm{d} r}{c}$ is the acoustic depth of the localised feature, $c$ the speed of sound, $r_{\mathrm{d}}$ the radial distance where the feature is located, $\omega_{n, \ell}$ the angular frequency of a mode with radial order $n$ and degree $\ell$, and $\phi$ a phase factor. This oscillatory signature has been extensively studied for the Sun in order to determine the extent of overshoot below the solar convection zone (Monteiro et al. 1994; Basu et al. 1994; Roxburgh \& Vorontsov 1994). It has been proposed earlier that this may also be used for distant stars to find the position of the base of the convective envelope or the second helium ionisation zone (Monteiro et al. 2000; Mazumdar \& Antia 2001; Roxburgh \& Vorontsov 2003; Ballot et al. 2004; Basu et al. 2004; Mazumdar 2005). Indeed, Miglio et al. (2010) have used the modulation of the frequency separations to determine the location of the second helium ionisation zone in a red giant star.

We present here the results of applying this technique for the first time to a main sequence solar-type star, HD 49933, observed by the CoRoT mission in 2007-08 (Baglin et al. 2006).

\section{The technique}

The oscillatory signal in the frequencies is quite weak and is embedded in the frequencies, together with a smooth trend arising from the regular variation of the sound speed in the stellar interior. It can be enhanced by using the second differences $\delta^{2} v(n, \ell)=v(n-1, \ell)-2 v(n, \ell)+v(n+1, \ell)$, instead of the frequencies themselves (see, e.g., Gough 1990; Basu et al. 1994; Mazumdar \& Antia 2001; Basu et al. 2004).

The acoustic depths of the base of the convective zone (BCZ) and the second helium ionisation zone (HIZ), $\tau_{\mathrm{BCZ}}$ and $\tau_{\mathrm{HIZ}}$, respectively, can be obtained from the data by fitting the second differences to a suitable function representing the oscillatory signals from these layers (Mazumdar \& Antia 2001). We follow Basu et al. (2004) in choosing the following functional form:

$$
\begin{aligned}
\delta^{2} v= & a_{0}+b_{0} \sin \left(4 \pi v \tau_{\mathrm{BCZ}}+\phi_{\mathrm{BCZ}}\right) \\
& +\left(c_{0}+c_{2} / v^{2}\right) \sin \left(4 \pi v \tau_{\mathrm{HIZ}}+\phi_{\mathrm{HIZ}}\right)
\end{aligned}
$$

where $a_{0}, b_{0}, c_{0}, c_{2}, \tau_{\mathrm{BCZ}}, \phi_{\mathrm{BCZ}}, \tau_{\mathrm{HIZ}}$, and $\phi_{\mathrm{HIZ}}$ are eight free parameters of fitting. Since the number of data points in the observed set of frequencies for HD 49933 is quite small, we optimise the number of free parameters to strike a balance between a fair representation of the oscillatory signal and a reasonable $\chi^{2}$. We find that the smooth component is fairly constant over the range of frequencies that we use, as is the amplitude of the signal from the BCZ. However, the amplitude of the signal from the HIZ varies more sharply with frequency, and thus requires at least one frequency-dependent term. We note that Basu et al. (2004) have shown that the exact form of the amplitudes of the oscillatory signal does not affect the results significantly. To test our results, we also fit a function suggested by Houdek \& Gough (2007) (simplified version of Eq. (22) in that paper, with 8 free parameters).

The fit is carried out through a non-linear $\chi^{2}$ minimisation, weighted by the errors in the data. The errors in the second differences are correlated, and this is taken care of by defining the $\chi^{2}$ using a covariance matrix. The effects of the errors are considered by producing 1000 realisations of the data, where the mean values of the frequencies are skewed by random errors corresponding to a normal distribution. The successful convergence 
of such a non-linear fitting procedure is somewhat dependent on the choice of reasonable initial guesses. To remove the effect of initial guesses affecting the final fitted parameters, we carry out the fit for multiple combinations of starting values. For each realisation, 100 random combinations of initial guesses are tried for fitting the function above and from among the resulting fits, the one that produces the minimum value of $\chi^{2}$ is accepted as the fit for that particular realisation.

The median value of each parameter for 1000 realisations is taken as its fitted value. The error in the parameter is estimated from the range of values covering $68 \%$ area about the median (corresponding to $1 \sigma$ error). As an example, the histogram of distribution of the parameters $\tau_{\mathrm{BCZ}}$ and $\tau_{\mathrm{HIZ}}$ over multiple realisations are illustrated in Fig. 1. Thus the quoted errors in these parameters reflect the width of these histograms on two sides of the median value.

The strength of the oscillatory signals are measured through the amplitudes of the respective oscillatory components in the frequencies themselves. These are denoted by $A_{\mathrm{BCZ}}$ and $A_{\mathrm{HIZ}}$ and estimated by averaging the amplitudes of the oscillatory components in Eq. (1) over the observed frequency range, and correcting for the scaling factor of $4 \sin ^{2}\left(\pi \tau / \tau_{0}\right)$ arising in the second differences (see Mazumdar \& Antia 2001). In the present study, we use the amplitudes only as an index of the significance of the detected depth of the acoustic glitches.

We tested the technique on the frequencies of a polytropic model, with a polytropic index of 1.5 , provided by ChristensenDalsgaard \& Mullan (1994). Since there are no sharp acoustic features in such a model, we do not expect to find any oscillatory pattern in the second differences of the frequencies. Indeed, if we attempt to fit a function of the form given by Eq. (1) to the second differences of this model, we only find a signal corresponding to half of the large separation, which is the periodicity at which the successive points due to $\ell=0$ or 2 and $\ell=1$ occur.

The range of acoustic depth that can be probed by this technique is limited. The smallest depth that can be fitted corresponds to the range in the observed frequencies, and is $\sim 350 \mathrm{~s}$. On the other hand, the sampling frequency of the data points determines the largest acoustic depth that can be fitted. Since the average large separation of HD 49933 is $85 \mu \mathrm{Hz}$, the upper limit on the acoustic depth that can be fitted is $\sim 2940 \mathrm{~s}$.

\section{Results}

\subsection{Fitting the CoRoT frequencies}

We use the second differences of the frequencies obtained by Benomar et al. (2009) to extract the acoustic depths of BCZ and HIZ of HD 49933. We ignore frequencies that have errors of more than $3 \mu \mathrm{Hz}$. This gives a total of $31 \mathrm{~s}$ differences to be used for $\ell=0,1,2$. We consider four different subsets of the data: (i) $\ell=0$ modes only (12 data points), (ii) $\ell=1$ modes only (16 data points), (iii) $\ell=0,1$ modes (28 data points), and (iv) $\ell=0,1,2$ modes (31 data points). This was done to test the consistency of the results when modes of different degrees are considered, especially since the $\ell=0$ and $\ell=2$ modes in the data have, on the average, higher errors than the $\ell=1$ modes. However, the number of data points in the first two cases are only marginally larger than the number of free parameters in the fitting function, and these cases are only used for testing.

The fits to the second differences of the mean frequencies and the distribution of the fitted acoustic depths of BCZ and HIZ for different realisations of the data are shown in Fig. 1. Although we fit 1000 realisations of the data, only those
Table 1. Results of fitting second differences of HD 49933 frequencies to Eq. (1).

\begin{tabular}{lcccc}
\hline \hline$\ell$ & $\tau_{\mathrm{BCZ}}(\mathrm{s})$ & $\tau_{\mathrm{HIZ}}(\mathrm{s})$ & $A_{\mathrm{BCZ}}(\mu \mathrm{Hz})$ & $A_{\mathrm{HIZ}}(\mu \mathrm{Hz})$ \\
\hline 0 & $1877_{-340}^{+273}$ & $847_{-253}^{+248}$ & $1.06_{-0.34}^{+0.59}$ & $1.92_{-0.70}^{+5.80}$ \\
1 & $1496_{-194}^{+438}$ & $812_{-79}^{+45}$ & $0.75_{-0.20}^{+0.24}$ & $1.47_{-0.29}^{+0.33}$ \\
0,1 & $1626_{-262}^{+327}$ & $806_{-61}^{+45}$ & $0.66_{-0.15}^{+0.18}$ & $1.40_{-0.22}^{+0.28}$ \\
$0,1,2$ & $1855_{-412}^{+173}$ & $794_{-68}^{+55}$ & $0.71_{-0.15}^{+0.19}$ & $1.32_{-0.31}^{+0.34}$ \\
\hline Model & 1998 & 882 & & \\
$0,1,2$ & $1982_{-586}^{+447}$ & $848_{-62}^{+61}$ & $0.47_{-0.13}^{+0.19}$ & $1.37_{-0.22}^{+0.24}$ \\
\hline
\end{tabular}

Notes. Median values of the acoustic depths and amplitudes and their errors are listed. The last two rows give the calculated and the fitted values of a representative stellar model of HD 49933.

realisations with $\tau_{\mathrm{BCZ}} \leq 2700 \mathrm{~s}$ are considered for reasons explained below. A fit to the data of subset (iv) above to the form suggested by Houdek \& Gough (2007) is also shown in Fig. 1. Table 1 lists the median values of the acoustic depths with estimated errors and the corresponding amplitudes of BCZ and HIZ signals with estimated errors for the four data subsets.

The acoustic depth of the HIZ obtained from the different sets are consistent within the errorbars. The amplitude of the HIZ oscillatory function is also significant at more than $2.5 \sigma$ level in each set.

However, the BCZ oscillatory signal is not so consistent among different sets. For all values of $\ell$, we get a peak in the histogram close to the value $\tau_{0} / 2 \sim 2900 \mathrm{~s}$, which corresponds to the Nyquist frequency. The occurrence of this peak could mean that the amplitude of the oscillatory function corresponding to $\mathrm{BCZ}$ is close to zero, so that the fitted sine wave has a periodicity equal to the natural spacing of the data points, i.e., the large separation. While this seems to be the case for $\ell=1$ modes, the second differences of the radial modes appear to have an actual oscillatory signal corresponding to this frequency! Thus this effect cannot be fully ascribed to the Nyquist effect. This is also borne out by the persistence of this peak even when we combine data with multiple values of $\ell$, which should not be the case (see, e.g., Mazumdar \& Antia 2001). We cannot find a satisfactory explanation for the origin of this periodicity in the radial modes, apart from an artifact introduced by the noise. To eliminate this artifact for all subsets, we discard the realisations for which the BCZ signal is fitted with an acoustic depth of around 2900 s. Few realisations yield $\tau_{\mathrm{BCZ}}$ values higher than the acoustic midpoint of the star ( 2900 s), and these are also discarded. Actually, we only consider those realisations for which $\tau_{\mathrm{BCZ}}<2700 \mathrm{~s}$, since the peak in the histogram around the acoustic midpoint has a typical spread of $200 \mathrm{~s}$ on either side of the peak. This results in reducing the number of valid realisations by nearly $30 \%$.

In all the cases, there is a peak in the histogram for $\tau_{\mathrm{BCZ}}$ around $1950 \mathrm{~s}$, which is most likely due to the base of the convective envelope in the star. For $\ell=0$ and $\ell=0,1,2$, this is the dominant peak of the histogram, while for $\ell=1$ and $\ell=0,1$, this is the secondary peak. For the last two cases, the dominant peak is centred on $1370 \mathrm{~s}$. If this value is to correspond to $\tau_{\mathrm{BCZ}}$ it would imply a very shallow convection zone in the star, which is an unlikely scenario given the expected mass and age of HD 49933. This peak cannot be associated with any artifact due to the sampling frequency, but on the other hand, we cannot assign any physical significance to it.

To understand the difference in the values of $\tau_{\mathrm{BCZ}}$ obtained for different $\ell$, we carried out the following test. For each case, 

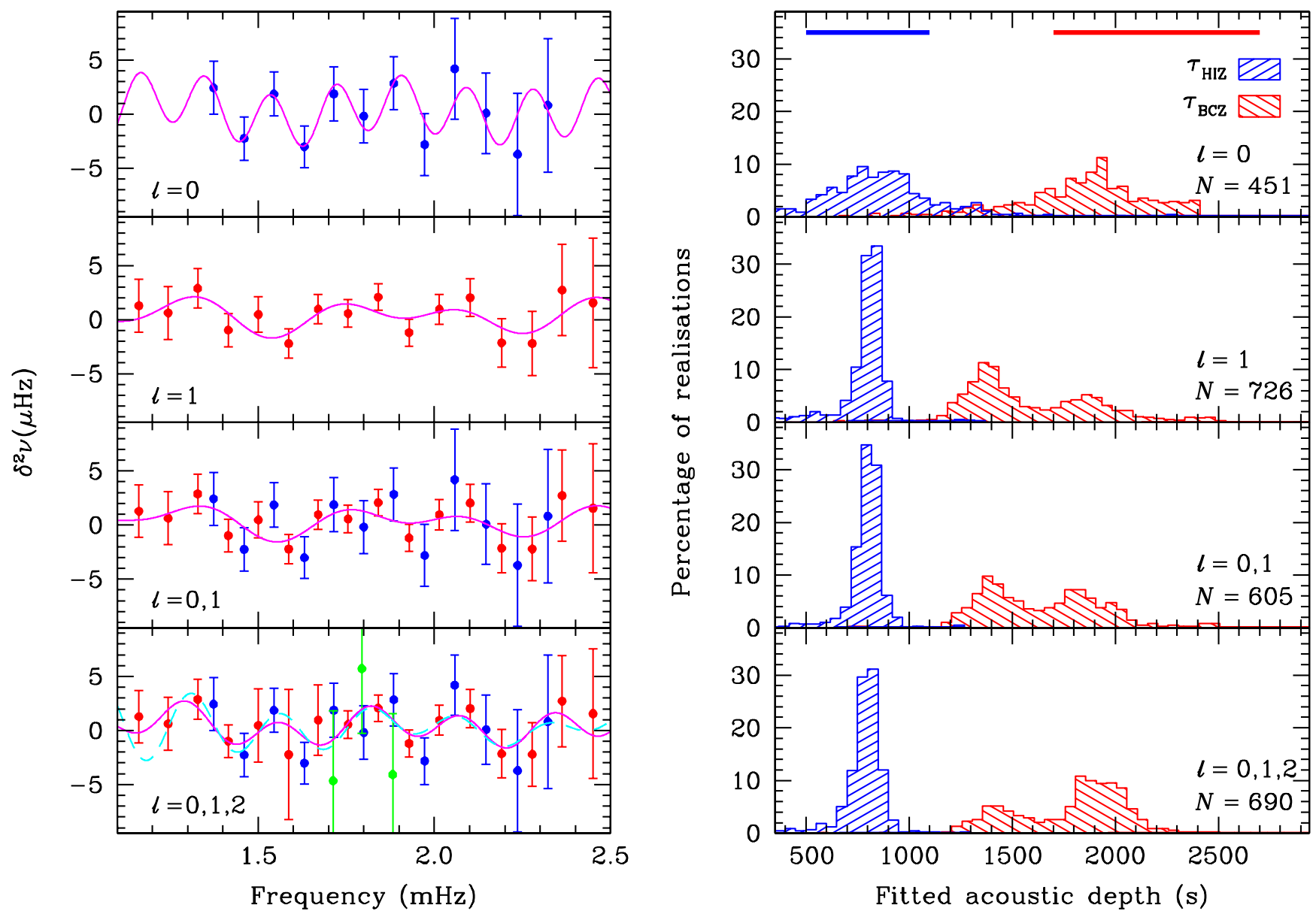

Fig. 1. Fits of Eq. (1) to the second differences of the mean CoRoT frequencies for HD 49933 and the histograms for the fitted values of $\tau_{\text {HIZ }}$ and $\tau_{\mathrm{BCZ}}$ for different realisations of the data for different subsets (corresponding to different choices of $\ell$ values). The second differences of the frequencies of $\ell=0$ (blue), $\ell=1$ (red) and $\ell=2$ (green) modes of HD 49933 and their fit to Eq. (1) (magenta curve) are shown in the left panel. The dotted cyan line in the bottom left panel shows the fit by the Houdek \& Gough (2007) form. The corresponding histograms of the fitted values of $\tau_{\mathrm{BCZ}}$ (in red) and $\tau_{\mathrm{HIZ}}$ (in blue) for different realisations are shown in the right panel. $N$ denotes the total number of valid realisations after rejecting fits with $\tau_{\mathrm{BCZ}}>2700 \mathrm{~s}$. The solid bands at the top of the right panel indicate the range of initial guesses for the two parameters in each fit.

we held the parameter $\tau_{\mathrm{BCZ}}$ in Eq. (1) fixed at a certain value, while allowing all other parameters to be free, and monitored the value of the reduced $\chi^{2}$ obtained by the resulting fit to the mean second differences. The fixed value of $\tau_{\mathrm{BCZ}}$ was changed continuously between $1000 \mathrm{~s}$ and $4800 \mathrm{~s}$ to find the minima in the reduced $\chi^{2}$ as this parameter varies. The $\chi^{2}$ is plotted as a function of $\tau_{\mathrm{BCZ}}$ in Fig. 2. We find that the $\ell=1$ modes show a deep minimum around $1370 \mathrm{~s}$, but only a slight inflexion around $1950 \mathrm{~s}$. In contrast, the location of the minimum and the inflexion are interchanged for the $\ell=0$ modes. When these modes are combined with the $\ell=2$ modes, we have minima at both the values. The third minimum around $2900 \mathrm{~s}$ for all cases has already been discussed. The almost mirrored shapes of the graphs above $\tau_{\mathrm{BCZ}}$ values of $2900 \mathrm{~s}$ merely illustrate the aliasing effect, as elaborated in Mazumdar \& Antia (2001).

\subsection{Fitting model frequencies}

We fit Eq. (1) to the second differences of frequencies calculated from a representative stellar model for the star HD 49933 as well. The model is generated with the CESAM2k evolution code (Morel \& Lebreton 2008) and uses standard physical inputs,

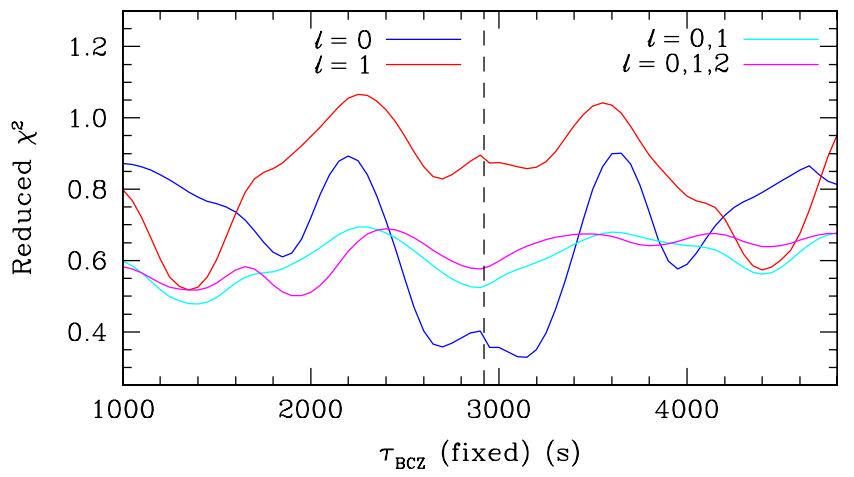

Fig. 2. The variation in $\chi^{2}$ with the adopted value of $\tau_{\mathrm{BCZ}}$ for four subsets of the CoRoT data on HD 49933 corresponding to different choices of the degree, $\ell$. The vertical dotted line shows the acoustic midpoint of the star.

including convective core overshoot, but no diffusive mixing. While we use the theoretical frequencies as the mean values in this case, we set the errors in the frequencies equal to the errors in the observed frequencies for the corresponding mode, in 


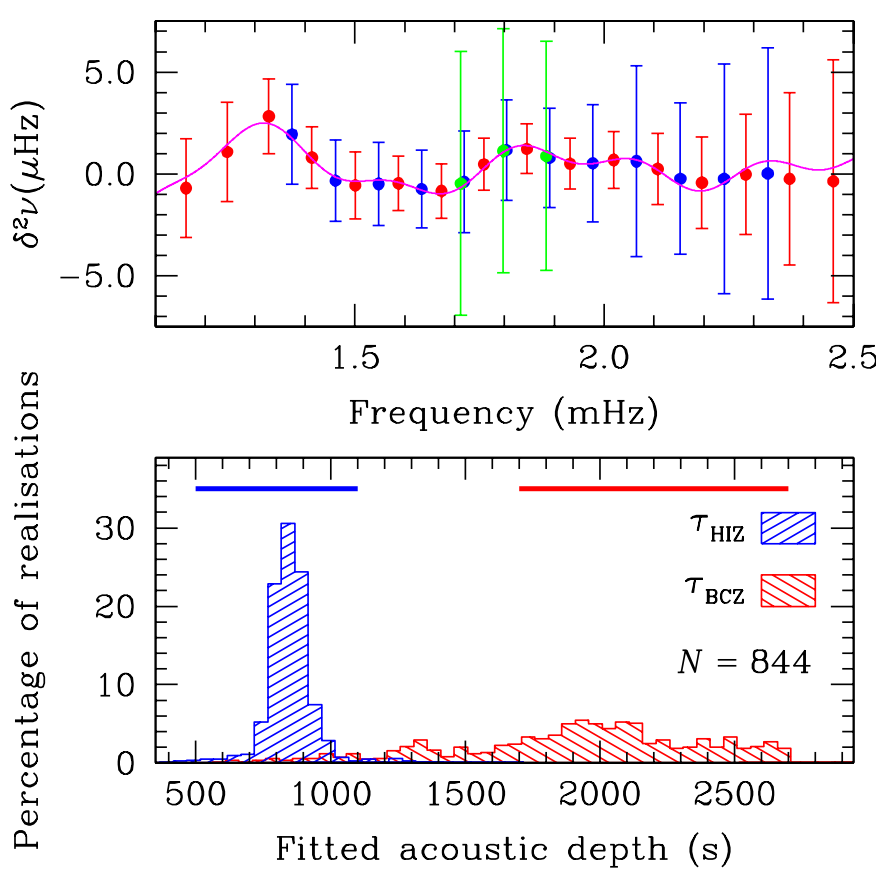

Fig. 3. Fits of Eq. (1) to the second differences of the frequencies of a theoretical model for HD 49933 (top panel) and the histograms for different realisations of the data for the fitted values of $\tau_{\mathrm{HIZ}}$ and $\tau_{\mathrm{BCZ}}$ (bottom panel). See Fig. 1 for explanations of colours and symbols.

order to mimic the uncertainties present in the data. The results of such a fit are displayed in Fig. 3. The model values, as well as the fitted median values of $\tau_{\mathrm{HIZ}}$ and $\tau_{\mathrm{BCZ}}$, are given in Table 1 .

We find that the median values of the acoustic depths of both $\mathrm{BCZ}$ and HIZ are recovered from the frequencies. However, the error in the value of $\tau_{\mathrm{BCZ}}$ is quite large owing to the flat nature of the histogram in Fig. 3. In contrast, the histogram of the HIZ signal is sharply peaked, so the estimated error in the median value of $\tau_{\mathrm{HIZ}}$ is also smaller. We conclude that the intrinsic oscillatory signal arising from the acoustic glitch at BCZ is quite weak, and it is difficult to extract this signal even with model frequencies. It is beyond the scope of this study to investigate why this signal is so weak, and it needs to be probed whether this is a common feature of all stars in the relevant part of the HR diagram. The HIZ signal, however, is strong and can easily be used to extract the acoustic depth of the second helium ionisation zone.

\section{Summary}

We have applied the method of determining the acoustic depth of layers of sharp variation in sound speed in stellar interiors from the oscillatory signal in frequencies to the CoRoT primary target HD 49933.

The oscillatory signal is made more pronounced in comparison to the smooth change in sound speed by using the second differences of the frequencies. We fit a function with two oscillatory components corresponding to the two major layers of sharp variation in sound speed, viz., the base of the convective envelope and the second helium ionisation zone. To prove that the technique does not produce artificial signatures of oscillatory signals when there are none, we tested it on the frequencies of a polytropic model where there are no real layers of sharp variation in the sound speed. In this case the only significant oscillatory signal corresponds to the uniform spacing of the frequencies, as expected.

We find that the oscillatory signal arising due to the second helium ionisation zone is quite strong in the CoRoT data of HD 49933. We estimated the acoustic depth of this layer to be $794_{-68}^{+55} \mathrm{~s}$. This is borne out by the frequencies of modes of all available degrees: $\ell=0,1,2$, when considered either separately or in combination. The acoustic depth of this layer in a representative stellar model is consistent with this value, and can also be easily extracted by our technique.

The oscillatory signal due to the base of the convective envelope is far weaker in the data, in comparison to the helium signal. Moreover, we find that the signal is not consistent between modes of different degrees. We find additional periodicities in the frequencies corresponding to different acoustic depths in modes of $\ell=0$ and $\ell=1$, for which we are unable to assign any physical acoustic feature inside the star. It seems likely that these are artifacts due to the errors in the data which have no physical relevance. Nevertheless, we do find an oscillatory signal corresponding to an acoustic depth of $1855_{-412}^{+173} \mathrm{~s}$ in the combined frequency set of different degrees, which is consistent with the position of the base of the convective envelope in a representative stellar model. This oscillatory signal is weak even in the model frequencies, and is fairly difficult to extract from the second differences. These results prove that the technique of extracting acoustic depths of sharp features inside a star can be successfully applied to real asteroseismic data.

Acknowledgements. CoRoT (Convection, Rotation and planetary Transits) space mission has been developed and is operated by the French Space agency CNES in collaboration with Austria, Belgium, Brazil, ESA's RSSd and Science Programmes, Germany, and Spain. A.M. acknowledges support from the National Initiative on Undergraduate Science (NIUS) undertaken by the Homi Bhabha Centre for Science Education - Tata Institute of Fundamental Research (HBCSE-TIFR), Mumbai, India.

\section{References}

Baglin, A., Auvergne, M., Barge, P., et al. 2006, ESA SP, 1306, 33 Ballot, J., Turck-Chièze, S., \& García, R. A. 2004, A\&A, 423, 1051 Basu, S., Antia, H. M., \& Narasimha, D. 1994, MNRAS, 267, 209 Basu, S., Mazumdar, A., Antia, H. M., \& Demarque, P. 2004, MNRAS, 350, 277 Benomar, O., Baudin, F., Campante, T. L., et al. 2009, A\&A, 507, L13 Christensen-Dalsgaard, J., \& Mullan, D. J. 1994, MNRAS, 270, 921 Gough, D. O. 1990, Progress of Seismology of the Sun and Stars, 367, 283 Houdek, G., \& Gough, D. O. 2007, MNRAS, 375, 861

Mazumdar, A. 2005, A\&A, 441, 1079

Mazumdar, A., \& Antia, H. M. 2001, A\&A, 377, 192

Miglio, A., Montalbán, J., Carrier, F., et al. 2010, A\&A, 520, L6 Monteiro, M. J. P. F. G., Christensen-Dalsgaard, J., \& Thompson, M. J. 1994, A\&A, 283, 247

Monteiro, M. J. P. F. G., Christensen-Dalsgaard, J., \& Thompson, M. J. 2000, MNRAS, 316, 165

Morel, P., \& Lebreton, Y. 2008, Ap\&SS, 316, 61

Roxburgh, I. W., \& Vorontsov, S. V. 1994, MNRAS, 268, 880

Roxburgh, I. W., \& Vorontsov, S. V. 2003, A\&A, 411, 215 